\title{
Sensitivity Analysis and Density Estimation for the Hobson-Rogers Stochastic Volatility Model
}

\author{
REIICHIRO KAWAI
}

\begin{abstract}
Monte Carlo estimators of sensitivity indices and the marginal density of the price dynamics are derived for the Hobson-Rogers stochastic volatility model. Our approach is based mainly upon the Kolmogorov backward equation by making full use of the Markovian property of the dynamics given the past information. Some numerical examples are presented with a GARCHlike volatility function and its extension to illustrate the effectiveness of our formulae together with a clear exhibition of the skewness and the heavy tails of the price dynamics.

Keywords: Asset price dynamics, density estimation, GARCH, Kolmogorov backward equation, Markov processes, Monte Carlo simulation, sensitivity analysis, stochastic volatility.

2000 Mathematics Subject Classification: 91B28, 62M05, 62G07, $65 \mathrm{C} 05$.
\end{abstract}

\section{Introduction}

The Hobson-Rogers stochastic volatility model [12] induces the complete market and the standard non-arbitrage pricing argument holds true just as in the Black-Scholes market, since no exogenous source of risk is introduced. This property is a superiority to other popular stochastic volatility models, such as the Heston model [11] and the SABR model [8]. Meanwhile, similarly to those stochastic volatility models, the Hobson-Rogers model is still capable of capturing the market volatility smiles. Due to those fine features, the model has attracted a significant attention from various aspects. For example, some empirical analyses are performed in Platania and Rogers [15] and in Foschi and Pascucci [5], while the calibration problems are considered in Foschi and Pascucci [5] and in Figà-Talamanca and Guerra [3]. The robustness of the model with respect to the data and the parameters is investigated in Hallulli and Vargiolu [10]. A generalization of the model is studied in Hubalek, Teichmann and Tompkins [13], while an extension to the framework of term-structure modeling is proposed in Chiarella and Kwon [2] and its dynamics is applied in the context of portfolio optimization in Hahn, Putschögl and Sass [9].

Published in International Journal of Theoretical and Applied Finance (2009) 12(3) 283-295.

*Email address: reiichiro_kawai@ybb.ne.jp. Postal address: Center for the Study of Finance and Insurance, Osaka University, Toyonaka, 560-8531, Japan. 
The aim of this study is to derive sensitivity indices of delta and gamma and marginal density estimation formulae of the price dynamics for the Hobson-Rogers stochastic volatility model. The importance and usefulness of the delta formula comes from the market completeness, since it implies the perfect delta-hedge of contingent claims. Meanwhile, our density estimation formula of logarithmic marginal provides one a convenient way to investigate its distribution skew and tails. Our approach is based upon the Kolmogorov backward equation by making full use of the Markovian property of the dynamics rather than the standard Malliavin integration-by-parts argument. In our framework, the volatility function may consist of two or more arguments so that the effect of higher-order offset processes (which will be defined below) can be incorporated in full. This is a major advantage to approaches taken in the existing literature. They impose the assumption of the volatility function consisting only of the first-order offset process so that the contingent claim premium is the solution of a three-dimensional Kolmogorov partial differential equation. Without the assumption, one is required to deal numerically with a higher dimensional Kolmogorov partial differential equation.

The rest of this paper is organized as follows. Section 2 recalls the Hobson-Rogers stochastic volatility model and its featuring properties. Section 3 presents main results of this paper. First, by imposing a mild assumption on the past information, we derive a derivative formula of the price dynamics (Lemma 3.2), which is a crucial building block for all our results. Two fundamental sensitivity indices, delta and gamma, are derived in the form of the expectation towards Monte Carlo simulation (Theorem 3.4 and 3.5). Finally, from the delta formula, we derive the marginal density estimation formulae of the dynamics and of its logarithm (Theorem 3.8). Some numerical results are presented in Section 4 to illustrate the effectiveness of our formulae. Finally, Section 5 concludes this study.

\section{Preliminaries}

Let us begin with general notations which will be used throughout the text. $\mathbb{N}$ is the collection of all positive integers. $\mathbb{E}_{\mathbb{Q}}[\cdot]$ indicates the expectation taken under a probability measure $\mathbb{Q}$. The restriction of a probability measure $\mathbb{Q}$ to the $\sigma$-field $\mathscr{F}$ is denoted by $\left.\mathbb{Q}\right|_{\mathscr{F}}$. Leb $(\cdot)$ indicates the Lebesgue measure. For $k \in \mathbb{N}, \partial_{k}$ indicates the partial derivative with respect to $k$-th argument. We denote by $C_{b}^{k}$ the class of $k$-time continuously differentiable functions with bounded derivatives, while the subscript $c$ in $C_{c}^{k}$ indicates the existence of a compact support. We denote by $\mathscr{G}(\mathscr{E})$ the $\sigma$-field generated by $\mathscr{E}$.

Let us review the Hobson-Rogers stochastic volatility model introduced in [12]. Define a log asset price dynamics $\left\{Z_{t}: t \in \mathbb{R}\right\}$ by

$$
Z_{t}:=\ln S_{t}
$$

and offset processes (of order $m \in\{0\} \cup \mathbb{N}$ ) $\left\{Y_{t}^{(m)}: t \in[0,+\infty)\right\}$ by

$$
Y_{t}^{(m)}:=\int_{0}^{+\infty} \lambda e^{-\lambda s}\left(Z_{t}-Z_{t-s}\right)^{m} d s
$$


For $\sigma: \mathbb{R}^{n} \mapsto \mathbb{R}_{+}$, the martingale asset price dynamics $\left\{S_{t}: t \geq 0\right\}$ is formulated via the stochastic differential equation

$$
d S_{t}=S_{t} \sigma\left(Y_{t}^{(1)}, \ldots, Y_{t}^{(n)}\right) d W_{t}
$$

In what follows, we will call the function $\sigma(\cdot)$ the volatility function. For ease in notation, we will write for fixed $n \in \mathbb{N}$,

$$
\sigma_{t}:=\sigma\left(Y_{t}^{(1)}, \ldots, Y_{t}^{(n)}\right)
$$

Next, define a filtration

$$
\mathscr{F}_{t}:=\mathscr{F}_{0} \otimes \mathscr{G}\left(\left\{Z_{s}: s \in[0, t]\right\}\right) \otimes\left(\otimes_{m=1}^{n} \mathscr{G}\left(\left\{Y_{s}^{(m)}: s \in[0, t]\right\}\right)\right) .
$$

We know from Lemma 3.1 [12] that if $\left\{Z_{t}: t \in(-\infty, 0]\right\}$ is $\mathscr{F}_{0}$-measurable, then the stochastic process $\left(S, Y^{(1)}, \cdots, Y^{(n)}\right)$ is Markovian, and that for $t \in[0,+\infty)$ and for $m=1, \ldots, n$,

$$
d Y_{t}^{(m)}=m Y_{t}^{(m-1)} d Z_{t}+\frac{m(m-1)}{2} Y_{t}^{(m-2)} d\langle Z\rangle_{t}-\lambda Y_{t}^{(m)} d t,\left.\quad \mathbb{P}\right|_{\mathscr{F}_{0}}-\text { a.s. }
$$

where $Y^{(-1)} \equiv 0$.

Notice that the induced market is complete since no additional source of randomness, other than $\left\{W_{t}: t \geq 0\right\}$ in (2.2), is introduced in the model. This implies that there is a unique preference-free premium for contingent claims, which can be perfectly replicated by the delta-hedge in view of the Brownian martingale representation.

In all the existing literature, the volatility function is assumed to consist only of one argument with the offset process of first order, that is, the form of $\sigma\left(Y_{t}^{(1)}\right)$. A practical reason for this assumption is that even the sole use of the first order offset process may yield a sufficient variety of volatility skews and smiles, while a more important reason comes from a theoretical point of view. By the Markovian property, the premium of a European contingent claim

$$
f\left(S_{t}, Y_{t}^{(1)}, T-t\right):=\mathbb{E}_{\mathbb{P}}\left[\Phi\left(S_{T}\right) \mid \mathscr{F}_{t}\right]
$$

follows the Feynman-Kac formula, where $\Phi$ is a suitable function. With the form of $\sigma\left(Y_{t}^{(1)}\right)$, the above $f$ is the solution of the three-dimensional Kolmogorov partial differential equation

$$
0=\left(-\lambda y \partial_{2} f-\partial_{3} f\right)+\left(-\frac{1}{2} \partial_{2} f+\frac{1}{2} s^{2} \partial_{1}^{2} f+\frac{1}{2} \partial_{2}^{2} f+s \partial_{1} \partial_{2} f\right) \sigma(y)^{2},
$$

with the boundary condition $f(s, y, 0)=\Phi(s)$. In particular, a numerical scheme of this equation is developed and investigated in Di Francesco and Pascucci [6]. Without the assumption of the single argument volatility function, the corresponding Kolmogorov partial differential equation would be too complicated to solve numerically, for example, by the finite difference method. 


\section{Main Results}

Let us begin with an assumption on the past information.

Standing Assumption 3.1. We assume that given $\mathscr{F}_{0}$, for $t \in(-\infty, 0]$,

$$
\frac{\partial S_{t}}{\partial S_{0}}=\frac{S_{t}}{S_{0}}
$$

The above assumption is not restrictive since it is equivalent in practice that we are given $\mathscr{F}_{0}$ to having all the past information at hand in the form of deterministic dynamics, which can be freely thought of as $S_{t}=S_{0} \beta_{t}$, where $\left\{\beta_{t}: t \in(-\infty, 0]\right\}$ is independent of $S_{0}$.

We next derive a derivative formula of the dynamics in the following lemma, which is crucial for discussions in what follows.

Lemma 3.2. Under Standing Assumption 3.1 we have that for $t \in(0,+\infty)$,

$$
\frac{\partial S_{t}}{\partial S_{0}}=\frac{S_{t}}{S_{0}},\left.\quad \mathbb{P}\right|_{\mathscr{F}_{0}} \text {-a.s. }
$$

Proof. First, by differentiating (2.1) at $t=0$ with respect to $S_{0}$, we get

$$
\frac{\partial Y_{0}^{(m)}}{\partial S_{0}}=\int_{0}^{+\infty} \lambda e^{\lambda s} \frac{\partial}{\partial S_{0}}\left(\ln S_{0}-\ln S_{-s}\right)^{m} d s=0,
$$

where the last equality follows from Assumption 3.1. Next, by differentiating (2.3) with respect to $S_{0}$, we obtain a linear stochastic differential equation

$$
d \frac{\partial Y_{t}}{\partial S_{0}}=A_{t} \frac{\partial Y_{t}}{\partial S_{0}} d W_{t}+B_{t} \frac{\partial Y_{t}}{\partial S_{0}} d t,\left.\quad \mathbb{P}\right|_{\mathscr{F}_{0}} \text {-a.s. }
$$

where

$$
\frac{\partial Y_{t}}{\partial S_{0}}:=\left(\frac{\partial Y_{t}^{(1)}}{\partial S_{0}}, \ldots, \frac{\partial Y_{t}^{(n)}}{\partial S_{0}}\right)^{\prime},
$$

and where $A_{t}$ and $B_{t}$ are suitable $\mathscr{F}_{t}$-measurable processes in $\mathbb{R}^{n \times n}$. From the initial conditions $\partial Y_{0}^{(m)} / \partial S_{0}=0$, it follows that $\partial Y_{t}^{(m)} / \partial S_{0}=0,\left.\mathbb{P}\right|_{\mathscr{F}_{0}}$-a.s. Hence, by differentiating (2.2), we get

$$
d \frac{\partial S_{t}}{\partial S_{0}}=\frac{\partial S_{t}}{\partial S_{0}} \sigma_{t} d W_{t}
$$

with the unit initial value. This indeed satisfies (2.2) and thus the proof is complete.

We will use the following notation; for $T>0$ and $0 \leq t_{1} \leq t_{2} \leq T$,

$$
L_{t_{1}, t_{2}}:=\int_{t_{1}}^{t_{2}} \sigma_{s}^{-1} d W_{s}
$$

Before proceeding to the presentation of main results, let us summarize all assumptions to be imposed on the volatility function $\sigma(\cdot)$. Note that unlike in all the existing literature, we do not impose assumptions on the volatility function to consists only of one argument nor to be smooth. 
Standing Assumption 3.3. The volatility function $\sigma: \mathbb{R}^{n} \mapsto \mathbb{R}$ takes values in a positive compact set, that is, there exist constants $a>0$ and $b \in[a,+\infty)$ such that $\sigma(\cdot) \in[a, b]$.

It is straightforward that the above assumption guarantees $\int_{0}^{T} \sigma_{t}^{-2} d t<+\infty,\left.\mathbb{P}\right|_{\mathscr{F}_{0}}-$ a.s., and $\mathbb{E}_{\mathbb{P}}\left[\int_{0}^{T} S_{t} d t \mid \mathscr{F}_{0}\right]<+\infty$.

\subsection{Delta}

We first consider the sensitivity of the European premium with respect to $S_{0}$, that is, Delta. The technique in the proof is well known (see, for example, Gikhman and Skorokhod [7]), and has already been applied to the sensitivity analysis in Cass and Friz [1] and Takeuchi [17]. As mentioned earlier, this sensitivity index is of particular importance in the sense that in the induced complete market, the contingent claim can be perfectly replicated by the delta-hedge.

Theorem 3.4. For $\Phi: \mathbb{R} \mapsto \mathbb{R}$ such that $\mathbb{E}_{\mathbb{P}}\left[\left|\Phi\left(S_{T}\right)\right|^{2}\right]<+\infty$, we have

$$
\frac{\partial}{\partial S_{0}} \mathbb{E}_{\mathbb{P}}\left[\Phi\left(S_{T}\right) \mid \mathscr{F}_{0}\right]=\frac{1}{S_{0} T} \mathbb{E}_{\mathbb{P}}\left[\Phi\left(S_{T}\right) L_{0, T} \mid \mathscr{F}_{0}\right]
$$

Proof. First, assume that $\Phi \in C_{b}^{2}(\mathbb{R} ; \mathbb{R})$. Define

$$
u(t, x):=\mathbb{E}_{\mathbb{P}}\left[\Phi\left(S_{T-t}\right) \mid S_{0}=x, \mathscr{F}_{0}\right] .
$$

Due to $\Phi \in C_{b}^{2}(\mathbb{R} ; \mathbb{R})$, the Ito formula reads

$$
\begin{aligned}
d u\left(t, S_{t}\right) & =\partial_{1} u\left(t, S_{t}\right) d t+\partial_{2} u\left(t, S_{t}\right) d S_{t}+\frac{1}{2} \partial_{2}^{2} u\left(t, S_{t}\right) d\langle S\rangle_{t} \\
& =\left[\partial_{1} u\left(t, S_{t}\right)+\frac{1}{2} \partial_{2}^{2} u\left(t, S_{t}\right) S_{t}^{2} \sigma_{t}^{2}\right] d t+\partial_{2} u\left(t, S_{t}\right) \sigma_{t} S_{t} d W_{t}
\end{aligned}
$$

By the Markov property of $\left(S, Y^{(1)}, \ldots, Y^{(n)}\right)$ and the time-homogeneity of $\sigma(\cdot)$, we have

$$
\mathbb{E}_{\mathbb{P}}\left[\Phi\left(S_{T-t}\right) \mid S_{0}=x, \mathscr{F}_{0}\right]=\mathbb{E}_{\mathbb{P}}\left[\Phi\left(S_{T}\right) \mid S_{t}=x,\left\{Y_{t}^{(m)}\right\}_{m=1, \ldots, n}, \mathscr{F}_{0}\right]
$$

and for $s \in[0, t]$,

$$
\begin{aligned}
\mathbb{E}_{\mathbb{P}}\left[u\left(t, S_{t}\right) \mid \mathscr{F}_{S}\right] & =\mathbb{E}_{\mathbb{P}}\left[\mathbb{E}_{\mathbb{P}}\left[\Phi\left(S_{T}\right) \mid S_{t},\left\{Y_{t}^{(m)}\right\}_{m=1, \ldots, n}, \mathscr{F}_{0}\right] \mid \mathscr{F}_{S}\right] \\
& =\mathbb{E}_{\mathbb{P}}\left[\Phi\left(S_{T}\right) \mid S_{s},\left\{Y_{s}^{(m)}\right\}_{m=1, \ldots, n}, \mathscr{F}_{0}\right] \\
& =u\left(s, S_{s}\right) .
\end{aligned}
$$

Hence, we get, for each $t \in(0, T]$,

$$
\partial_{1} u\left(t, S_{t}\right)+\frac{1}{2} \partial_{2}^{2} u\left(t, S_{t}\right) S_{t}^{2} \sigma_{t}^{2}=0,\left.\quad \mathbb{P}\right|_{\mathscr{F}_{0}}-\text { a.s. }
$$


It then follows that

$$
\begin{aligned}
\Phi\left(S_{T}\right) & =\mathbb{E}_{\mathbb{P}}\left[\Phi\left(S_{T}\right) \mid \mathscr{F}_{0}\right]+\int_{0}^{T} \partial_{2} u\left(s, S_{s}\right) \sigma_{s} S_{s} d W_{s} \\
& =\mathbb{E}_{\mathbb{P}}\left[\Phi\left(S_{T}\right) \mid \mathscr{F}_{0}\right]+S_{0} \int_{0}^{T} \frac{\partial}{\partial S_{0}} u\left(s, S_{s}\right) \sigma_{s} d W_{s},\left.\quad \mathbb{P}\right|_{\mathscr{F}_{0}} \text {-a.s. },
\end{aligned}
$$

where the last equality holds by Lemma 3.2

Next, it follows from Standing Assumption 3.3 that $\mathbb{E}_{\mathbb{P}}\left[L_{0, T}^{2} \mid \mathscr{F}_{0}\right]<+\infty$. Hence, by the ItoWiener isometry, we have

$$
\begin{aligned}
\mathbb{E}_{\mathbb{P}}\left[\Phi\left(S_{T}\right) L_{0, T} \mid \mathscr{F}_{0}\right] & =S_{0} \mathbb{E}_{\mathbb{P}}\left[\int_{0}^{T} \frac{\partial}{\partial S_{0}} u\left(t, S_{t}\right) d t \mid \mathscr{F}_{0}\right] \\
& =S_{0} \int_{0}^{T} \mathbb{E}_{\mathbb{P}}\left[\frac{\partial}{\partial S_{0}} u\left(t, S_{t}\right) \mid \mathscr{F}_{0}\right] d t \\
& =S_{0} \int_{0}^{T} \frac{\partial}{\partial S_{0}} \mathbb{E}_{\mathbb{P}}\left[u\left(t, S_{t}\right) \mid \mathscr{F}_{0}\right] d t \\
& =S_{0} T \frac{\partial}{\partial S_{0}} \mathbb{E}_{\mathbb{P}}\left[\Phi\left(S_{T}\right) \mid \mathscr{F}_{0}\right],
\end{aligned}
$$

where the first line to the second is verified by the Fubini theorem with the differentiability of $u(t, \cdot)$, where the second to the third is verified by the dominated convergence theorem with the assumption $\mathbb{E}_{\mathbb{P}}\left[\left|\Phi\left(S_{T}\right)\right|^{2}\right]<+\infty$ and with the Cauchy-Schwartz inequality, and where the last equality holds by the Markov property of $\left(S, Y^{(1)}, \ldots, Y^{(n)}\right)$.

Finally, the smoothness assumption of $\Phi$ can be removed by standard density arguments, which completes the proof.

\subsection{Gamma}

We next derive a Monte Carlo formula for Gamma, that is, the delta of the delta. From a theoretical point of view, this index is not very important due to the perfect delta-hedge, while it is still of great importance in practical discretization situations. Moreover, for its derivation, we follow the time interval separation technique again of [1] and [17], which may be of independent interest.

Theorem 3.5. Fix $T^{\prime} \in(0, T)$, and let $\Phi: \mathbb{R} \mapsto \mathbb{R}$ be such that $\mathbb{E}_{\mathbb{P}}\left[\left|\Phi\left(S_{T}\right)\right|^{2}\right]<+\infty$. Then, we have

$$
\frac{\partial^{2}}{\partial S_{0}^{2}} \mathbb{E}_{\mathbb{P}}\left[\Phi\left(S_{T}\right) \mid \mathscr{F}_{0}\right]=\frac{1}{S_{0}^{2} T^{\prime}\left(T-T^{\prime}\right)} \mathbb{E}_{\mathbb{P}}\left[\Phi\left(S_{T}\right) L_{0, T^{\prime}} L_{T^{\prime}, T} \mid \mathscr{F}_{0}\right]-\frac{1}{S_{0}} \frac{\partial}{\partial S_{0}} \mathbb{E}_{\mathbb{P}}\left[\Phi\left(S_{T}\right) \mid \mathscr{F}_{0}\right] .
$$

Proof. As in the proof of Theorem 3.4, we can first impose the assumption $\Phi \in C_{b}^{3}$, which is to be removed later by a density argument. By (3.1), we have that

$$
\mathbb{E}_{\mathbb{P}}\left[\Phi\left(S_{T}\right) \mid \mathscr{F}_{T^{\prime}}\right]=\mathbb{E}_{\mathbb{P}}\left[\Phi\left(S_{T}\right) \mid \mathscr{F}_{0}\right]+S_{0} \int_{0}^{T^{\prime}} \frac{\partial}{\partial S_{0}} u\left(s, S_{S}\right) \sigma_{s} d W_{s},\left.\quad \mathbb{P}\right|_{\mathscr{F}_{0}} \text { a.s. }
$$


By differentiating this, we get

$$
\begin{aligned}
\mathbb{E}_{\mathbb{P}}\left[\frac{\partial}{\partial S_{0}} \Phi\left(S_{T}\right) \mid \mathscr{F}_{T^{\prime}}\right]=\mathbb{E}_{\mathbb{P}} & {\left[\frac{\partial}{\partial S_{0}} \Phi\left(S_{T}\right) \mid \mathscr{F}_{0}\right] } \\
& +\int_{0}^{T^{\prime}} \frac{\partial}{\partial S_{0}} u\left(s, S_{s}\right) \sigma_{s} d W_{s}+S_{0} \int_{0}^{T^{\prime}} \frac{\partial^{2}}{\partial S_{0}^{2}} u\left(s, S_{s}\right) \sigma_{s} d W_{s},\left.\quad \mathbb{P}\right|_{\mathscr{F}_{0}} \text {-a.s. }
\end{aligned}
$$

where the passages to the limit hold by the dominated convergence theorem with the twicedifferentiability of $\Phi$. By the Ito-Wiener isometry, we get

$$
\begin{aligned}
\mathbb{E}_{\mathbb{P}}\left[\mathbb{E}_{\mathbb{P}}\left[\frac{\partial}{\partial S_{0}} \Phi\left(S_{T}\right) \mid \mathscr{F}_{T^{\prime}}\right] L_{0, T^{\prime}} \mid \mathscr{F}_{0}\right] & =\mathbb{E}_{\mathbb{P}}\left[\int_{0}^{T^{\prime}} \frac{\partial}{\partial S_{0}} u\left(s, S_{s}\right) d s \mid \mathscr{F}_{0}\right]+\mathbb{E}_{\mathbb{P}}\left[\int_{0}^{T^{\prime}} \frac{\partial^{2}}{\partial S_{0}^{2}} u\left(s, S_{s}\right) d s \mid \mathscr{F}_{0}\right] \\
& =T^{\prime} \frac{\partial}{\partial S_{0}} \mathbb{E}_{\mathbb{P}}\left[\Phi\left(S_{T}\right) \mid \mathscr{F}_{0}\right]+S_{0} T^{\prime} \frac{\partial^{2}}{\partial S_{0}^{2}} \mathbb{E}_{\mathbb{P}}\left[\Phi\left(S_{T}\right) \mid \mathscr{F}_{0}\right],
\end{aligned}
$$

where the last equality holds by the Fubini theorem, by the dominated convergence theorem, and by the Markov property of $\left(S, Y^{(1)}, \ldots, Y^{(n)}\right)$. Finally, the above left hand side proceeds

$$
\begin{aligned}
\mathbb{E}_{\mathbb{P}}\left[\mathbb{E}_{\mathbb{P}}\left[\frac{\partial}{\partial S_{0}} \Phi\left(S_{T}\right) \mid \mathscr{F}_{T^{\prime}}\right] L_{0, T^{\prime}} \mid \mathscr{F}_{0}\right] & =\mathbb{E}_{\mathbb{P}}\left[\frac{\partial}{\partial S_{0}} \mathbb{E}_{\mathbb{P}}\left[\Phi\left(S_{T}\right) \mid \mathscr{F}_{T^{\prime}}\right] L_{0, T^{\prime}} \mid \mathscr{F}_{0}\right] \\
& =\mathbb{E}_{\mathbb{P}}\left[\frac{\partial S_{T^{\prime}}}{\partial S_{0}} \frac{\partial}{\partial S_{T^{\prime}}} \mathbb{E}_{\mathbb{P}}\left[\Phi\left(S_{T}\right) \mid \mathscr{F}_{T^{\prime}}\right] L_{0, T^{\prime}} \mid \mathscr{F}_{0}\right] \\
& =\frac{1}{S_{0}\left(T-T^{\prime}\right)} \mathbb{E}_{\mathbb{P}}\left[\mathbb{E}_{\mathbb{P}}\left[\Phi\left(S_{T}\right) L_{T^{\prime}, T} \mid \mathscr{F}_{T^{\prime}}\right] L_{0, T^{\prime}} \mid \mathscr{F}_{0}\right] \\
& =\frac{1}{S_{0}\left(T-T^{\prime}\right)} \mathbb{E}_{\mathbb{P}}\left[\Phi\left(S_{T}\right) L_{0, T^{\prime}} L_{T^{\prime}, T} \mid \mathscr{F}_{0}\right],
\end{aligned}
$$

where the third equality holds by Lemma 3.2 and Theorem 3.4 . The proof is complete.

Remark 3.6. In the above formula, the choice of the time separation point $T^{\prime} \in(0, T)$ is arbitrary. It is a natural guess that the former part $L_{0, T^{\prime}}$ is very unstable if $T^{\prime}$ is extremely close to zero, while setting $T^{\prime}$ very close to $T$ makes the latter component $L_{T^{\prime}, T}$ instead very volatile. It seems that this conjecture is correct, as will be seen numerically in Section 4 .

Remark 3.7. One might have wondered if the standard Malliavin integration-by-parts argument leads to the above formulae. To see why not, observe first that for $u \in[0, T]$,

$$
D_{u} S_{T}=S_{T}\left[\sigma_{u}+\int_{0}^{T} D_{u} \sigma_{s} d W_{s}-\int_{0}^{T} \sigma_{s} D_{u} \sigma_{s} d s\right],\left.\quad \mathbb{P}\right|_{\mathscr{F}_{0}}-a . s .
$$

Clearly, this approach does not yield the same Greeks formulae, unless $D_{u} \sigma . \equiv 0,\left.\mathbb{P}\right|_{\mathscr{F}_{0}}$-a.s., while such an assumption is evidently unrealistic. It is indeed not very difficult to imagine that the derivation by means of the Malliavin integration-by-parts ends up with very intricate ones. 


\subsection{Density Estimation}

The marginal distribution of the dynamics has been investigated in the form of its Laplace transform in Sekine [16] with a linear volatility function $\sigma(y)=\alpha y, \alpha>0$. Without such a convenient choice of the volatility function, it seems very difficult to discuss such explicit information of the marginal distribution.

Here, we take a different approach of unbiased density estimation, again without imposing any specific structures on the volatility function. Our key tool is the delta formula of Theorem 3.4

Theorem 3.8. A density function $p_{\mathbb{P}}\left(x \mid \mathscr{F}_{0}\right)$ of $S_{T}$ at $x>0$ under the probability measure $\left.\mathbb{P}\right|_{\mathscr{F}_{0}}$ is given by

$$
p_{\mathbb{P}}\left(x \mid \mathscr{F}_{0}\right)=\frac{1}{x T} \mathbb{E}_{\mathbb{P}}\left[\mathbb{1}\left(S_{T}>x\right) L_{0, T} \mid \mathscr{F}_{0}\right] .
$$

Moreover, a density function $q_{\mathbb{P}}\left(x \mid \mathscr{F}_{0}\right)$ of $\ln \left(S_{T} / S_{0}\right)$ at $x \in \mathbb{R}$ under $\left.\mathbb{P}\right|_{\mathscr{F}_{0}}$ is given by

$$
q_{\mathbb{P}}\left(x \mid \mathscr{F}_{0}\right)=\frac{1}{T} \mathbb{E}_{\mathbb{P}}\left[\mathbb{1}\left(S_{T}>S_{0} e^{x}\right) L_{0, T} \mid \mathscr{F}_{0}\right]
$$

Proof. Due to Assumption 3.3, we can define a probability measure $\left.\mathbb{Q}\right|_{\mathscr{F} 0}$ via the Radon-Nikodym derivative

$$
\left.\frac{d \mathbb{Q}}{d \mathbb{P}}\right|_{\mathscr{F}_{t}}:=\frac{S_{t}}{S_{0}}, \quad t \in[0, T],\left.\quad \mathbb{P}\right|_{\mathscr{F}_{0}} \text {-a.s. }
$$

Let $\Phi(y)$ be in $C_{c}^{\infty}(\mathbb{R} ; \mathbb{R})$ such that $\Phi(y)=0$ if $y<x-h$ and $\Phi(y)=1$ if $y>x+h$, for $h>0$. In view of Lemma 3.2, the delta formula of Theorem 3.4 can be rewritten as

$$
\mathbb{E}_{\mathbb{Q}}\left[\Phi^{\prime}\left(S_{T}\right) \mid \mathscr{F}_{0}\right]=\frac{1}{S_{0} T} \mathbb{E}_{\mathbb{P}}\left[\Phi\left(S_{T}\right) L_{0, T} \mid \mathscr{F}_{0}\right]
$$

By standard approximation arguments, letting $h \downarrow 0$ yields a density function $p_{\mathbb{Q}}$ of $S_{T}$ at $x>0$ under $\left.\mathbb{Q}\right|_{\mathscr{F}_{0}}$ as

$$
p_{\mathbb{Q}}\left(x \mid \mathscr{F}_{0}\right)=\frac{1}{S_{0} T} \mathbb{E}_{\mathbb{P}}\left[\mathbb{1}\left(S_{T}>x\right) L_{0, T} \mid \mathscr{F}_{0}\right]
$$

Then, for $g \in C_{b}\left(\mathbb{R}_{+} ; \mathbb{R}\right)$, we have

$$
\mathbb{E}_{\mathbb{Q}}\left[g\left(S_{T}\right) \mid \mathscr{F}_{0}\right]=\mathbb{E}_{\mathbb{P}}\left[\left.\frac{d \mathbb{Q}}{d \mathbb{P}}\right|_{\mathscr{F}_{T}} g\left(S_{T}\right) \mid \mathscr{F}_{0}\right]=\mathbb{E}_{\mathbb{P}}\left[\frac{S_{T}}{S_{0}} g\left(S_{T}\right) \mid \mathscr{F}_{0}\right]=\int_{0}^{+\infty} g(x) \frac{x}{S_{0}} p_{\mathbb{P}}\left(x \mid \mathscr{F}_{0}\right) d x,
$$

which yields the first formula. Finally, the identity $q_{\mathbb{P}}\left(x \mid \mathscr{F}_{0}\right)=S_{0} e^{x} p_{\mathbb{P}}\left(S_{0} e^{x} \mid \mathscr{F}_{0}\right)$ leads directly to the second formula. The proof is complete.

Remark 3.9. For two reasons, the above density estimation formulae outperforms the standard kernel density estimation, for example,

$$
\widehat{q}_{\mathbb{P}}^{h}\left(x \mid \mathscr{F}_{0}\right):=\frac{1}{h} \mathbb{E}_{\mathbb{P}}\left[K\left(\frac{x-\ln \left(S_{T} / S_{0}\right)}{h}\right) \mid \mathscr{F}_{0}\right],
$$


where $K$ is a suitable kernel and where $h$ is the bandwidth. First, the kernel density estimation is only asymptotically unbiased as $h \downarrow 0$, while in reality $h$ can never be taken zero. On the other hand, our density estimators are intrinsically unbiased. Second, in the kernel density estimation, Monte Carlo summands provide almost no contribution to the convergence of Monte Carlo simulation unless realizations are very close to $x$; this problem turns out to be very serious when we set the bandwidth $h$ to be extremely small. In our formulae, meanwhile, all the Monte Carlo realizations with $S_{T}>S_{0} e^{x}$ (or with $S_{T}>x$ for the estimation of $p_{\mathbb{P}}$ ) make an equal contribution. We can see that the probability of the event $S_{T}>S_{0} e^{x}$ should not be very small unless $x$ is extremely large. For such an extreme case, it would be an effective approach to make use of the importance sampling variance reduction method by transforming the probability measure so that the realizations of $\ln \left(S_{T} / S_{0}\right)$ tend to generate larger values.

The following result is a direct consequence of the above density formula and should be useful in particular for the investigation of the tail probabilities.

Corollary 3.10. For $-\infty \leq a<b<+\infty$, we have

$$
\mathbb{P}\left(\ln \left(S_{T} / S_{0}\right) \in(a, b] \mid \mathscr{F}_{0}\right)=\frac{1}{T} \mathbb{E}_{\mathbb{P}}\left[\operatorname{Leb}\left(\left(-\infty, \ln \left(S_{T} / S_{0}\right)\right] \cap(a, b]\right) L_{0, T} \mid \mathscr{F}_{0}\right] .
$$

Proof. This is straightforward by

$$
\int_{a}^{b} q_{\mathbb{P}}\left(x \mid \mathscr{F}_{0}\right) d x=\frac{1}{T} \mathbb{E}_{\mathbb{P}}\left[\int_{a}^{b} \mathbb{1}\left(S_{T}>S_{0} e^{x}\right) d x L_{0, T} \mid \mathscr{F}_{0}\right],
$$

where the Fubini theorem is applied.

Remark 3.11. A similar superiority in the density estimation holds in this probability estimation. The Monte Carlo summands of our formula is zero only when $\ln \left(S_{T} / S_{0}\right)<a$, while in the standard probability estimator $\mathbb{E}_{\mathbb{P}}\left[\mathbb{1}\left(\ln \left(S_{T} / S_{0}\right) \in(a, b]\right) \mid \mathscr{F}_{0}\right]$, the summands are zero unless $\ln \left(S_{T} / S_{0}\right) \in$ $(a, b]$. It is not easy to adjust the probability measure transformation in a way that many realizations of $\ln \left(S_{T} / S_{0}\right)$ fall in the interval $(a, b]$ for the importance sampling variance reduction. One possible approach is the use of adaptive Monte Carlo variance reduction with stochastic approximation. (See, for example, Kawai [14].)

\section{Numerical Illustration}

Let us first compare the convergence of our Greeks formulae in Monte Carlo simulation to that of finite difference estimation, that is,

$$
\begin{aligned}
(\text { Delta }) & :=\frac{\mathbb{E}_{\mathbb{P}}\left[\Phi\left(S_{T}\right) \mid(1+\varepsilon) S_{0}, \mathscr{F}_{0}\right]-\mathbb{E}_{\mathbb{P}}\left[\Phi\left(S_{T}\right) \mid(1-\varepsilon) S_{0}, \mathscr{F}_{0}\right]}{2 \varepsilon S_{0}}, \\
(\text { Gamma }) & :=\frac{\mathbb{E}_{\mathbb{P}}\left[\Phi\left(S_{T}\right) \mid(1+\varepsilon) S_{0}, \mathscr{F}_{0}\right]-\mathbb{E}_{\mathbb{P}}\left[\Phi\left(S_{T}\right) \mid S_{0}, \mathscr{F}_{0}\right]+\mathbb{E}_{\mathbb{P}}\left[\Phi\left(S_{T}\right) \mid(1-\varepsilon) S_{0}, \mathscr{F}_{0}\right]}{\left(\varepsilon S_{0}\right)^{2}},
\end{aligned}
$$


with a slight abuse of notation. We fix $T=1, S_{0}=100$, and $\lambda=5$ in (2.3). Here, we only take into account $[-T, 0)$ of the past information. This restriction does not affect numerical results much so long as the decay rate $\lambda$ is set sufficiently high. We set the past dynamics $\left\{S_{t}: t \in[-T, 0]\right\}$ in a suitable manner such that $Y_{0}^{(1)}=0$. The next important setup is the volatility function. We use the one considered in [12, 4, 10] with the form of $\sigma_{1}\left(y_{1}\right):=\eta_{1}\left(1+\eta_{2} y_{1}^{2}\right)^{1 / 2} \wedge N$ with some large constant $N$ and positive $\eta_{1}, \eta_{2}$. Clearly, this is designed in the spirit of the discrete-time ARCH and GARCH to express the market consensus that large movements of the asset price dynamics in the past induce higher future volatility. To clearly illustrate the effectiveness of our Greeks formulae,

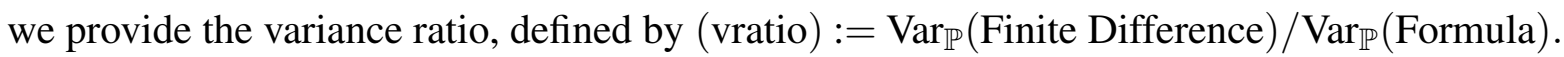

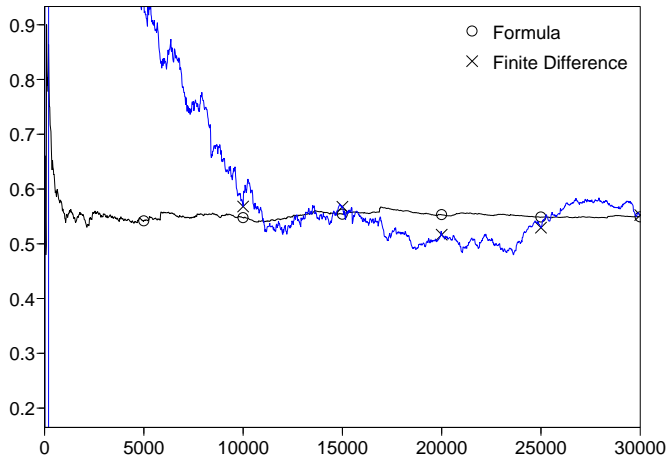

Delta $($ vratio $=210)$

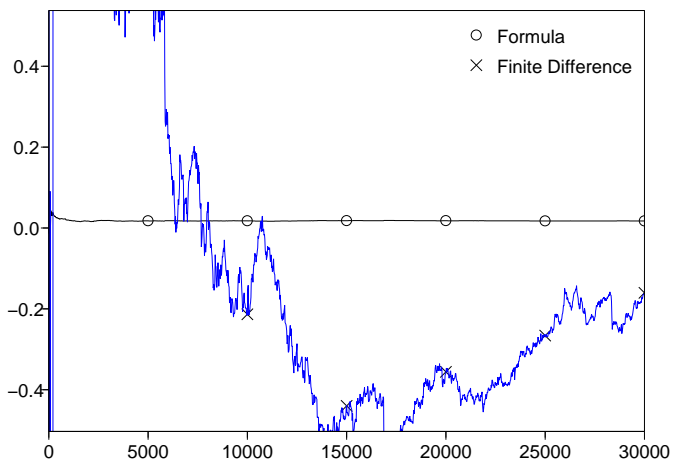

$\operatorname{Gamma}\left(T^{\prime}=T / 2\right.$, vratio $\left.=220,178\right)$

Figure 1: Monte Carlo convergence with $\Phi(x)=\left(x-S_{0}\right)_{+}$and $\varepsilon=1 \mathrm{e}-2$.

As mentioned in Remark 3.6, let us present how the choice of the time separation point $T^{\prime} \in$ $(0, T)$ changes the variance of the gamma estimator. Results should be different for different setups, while as Table 1 indicates, we conjecture that $T^{\prime}$ should better be chosen to be somewhere in the fairly middle of the interval.

\begin{tabular}{c||rrrrrrrrr}
$T^{\prime}$ & $0.1 T$ & $0.2 T$ & $0.3 T$ & $0.4 T$ & $0.5 T$ & $0.6 T$ & $0.7 T$ & $0.8 T$ & $0.9 T$ \\
\hline vratio $(\mathrm{e}+3)$ & 271 & 216 & 189 & 182 & 195 & 356 & 378 & 427 & 667
\end{tabular}

Table 1: Variance of the gamma estimator with different time separation points.

We are next concerned with the density $q_{\mathbb{P}}\left(x \mid \mathscr{F}_{0}\right)$ of $\ln \left(S_{T} / S_{0}\right)$. Together with the aforementioned volatility function $\sigma_{1}$, we also examine its extension with the form of $\sigma_{2}\left(y_{1}, y_{2}, y_{3}\right):=$ $\eta_{1}\left(1+\max \left(0, \eta_{2} y_{1}^{2}+\eta_{3} y_{3}\right)\right)^{1 / 2} \wedge N$, with a positive $\eta_{3}$, to illustrate that our framework may work with the volatility function of two or more arguments. By using the third-order offset process $\left\{Y_{t}^{(3)}: t \geq 0\right\}$, we intend to reflect the trend of price dynamics and to express the well known negative correlation between volatility and price dynamics. We present in Figure 2 the results of density 
estimation for the both volatility functions $\sigma_{1}$ and $\sigma_{2}$ based upon 3e+4 Monte Carlo realizations of $\ln \left(S_{T} / S_{0}\right)$. For clear illustration, we indicate by o the normalized histogram of $\ln \left(S_{T} / S_{0}\right)$ based upon the same $3 e+4$ realizations. In the middle figures we plot the implied volatility smile induced by the obtained densities, while we give QQ-plot in the bottom figures to visualize the heavy tails of the densities. As clearly indicated in the volatility smile and the QQ-plot, the introduction of the third-order offset process fattens the left marginal tail. Let us finally add to mention that our formula is capable of working with various forms of the volatility function. For example, an EGARCH-like formulation $\sigma_{3}(y)=\eta_{1} \exp \left(\eta_{2} y^{2}\right) \wedge N$ can yield similar forms of volatility smile and skew.

\section{Conclusion}

In this paper, we have derived Greeks formulae, of delta and gamma, and marginal density estimation formulae on the Hobson-Rogers stochastic volatility model. In principle, our approach is based upon the Kolmogorov backward equation by making full use of the Markovian property of the dynamics rather than the standard Malliavin integration-by-parts argument. Unlike the Kolmogorov partial differential equation approach in the existing literature, the volatility function in our framework may easily consist of two or more arguments so that the effect of higher-order offset processes can be incorporated in full. Our density estimation formula of logarithmic marginal provides one a convenient way to investigate distribution skew and tails. Numerical results indicate the effectiveness of our formulae and that the model can well create the skewness and the heavy tails of the marginal of the price dynamics. Finally, future research includes further numerical investigation with various volatility functions and parameter calibration to market data via an effective use of our density estimation formulae.

\section{Acknowledgements}

The author is grateful to an anonymous referee for a careful reading and several valuable suggestions. He would like to thank Arturo Kohatsu-Higa and Atsushi Takeuchi for fruitful and stimulating discussions.

\section{References}

[1] Cass, T., Friz, P. (2007) The Bismut-Elworthy-Li formula for jump-diffusions and applications to Monte Carlo methods in finance, Preprint available at arXiv:math/0604311v3.

[2] Chiarella, C., Kwon, K. (2000) A complete Markovian stochastic volatility model in the HJM framework, Asia-Pacific Financial Markets, 7(4) 293-304. 
[3] Figà-Talamanca, G., Guerra, M.L. (2006) Fitting prices with a complete model, Journal of Banking and Finance, 30(1) 247-258.

[4] Foschi, P., Pascucci, A. (2007) Calibration of the Hobson \& Rogers model: empirical tests, Preprint.

[5] Foschi, P., Pascucci, A. (2008) Path dependent volatility, Decisions in Economics and Finance, 31(1) 13-32.

[6] Di Francesco, M., Pascucci, A. (2004) On the complete model with stochastic volatility by Hobson and Rogers, Proceedings of The Royal Society A, 2051(8) 3327-3338.

[7] Gikhman, I.I., Skorokhod, A.V. (1972) Stochastic Differential Equations, Springer-Verlag.

[8] Hagan, P.S., Kumar, D., Lesniewski, A.S., Woodward, D.E. (2002) Managing smile risk, Wilmott Magazine, 1, 84-108.

[9] Hahn, M., Putschögl, W., Sass, J. (2007) Portfolio optimization with non-constant volatility and partial information, Brazilian Journal of Probability and Statistics, 21(1) 27-61.

[10] Hallulli, V., Vargiolu, T. (2007) Robustness of the Hobson-Rogers model with respect to the offset function, Proceedings of the Ascona '05 Seminar on Stochastic Analysis, Random Fields and Applications, 469-492, R. C. Dalang, M. Dozzi, F. Russo, editors, Birkhäuser.

[11] Heston, S. (1993) A closed-form solution for options with stochastic volatility with applications to bond and currency options, Review of Financial Studies, 6(2) 327-343.

[12] Hobson, D.G., Rogers, L.C.G. (1998) Complete models with stochastic volatility, Mathematical Finance, 8(1) 27-48.

[13] Hubalek, F., Teichmann, J., Tompkins, R. (2004) Flexible complete models with stochastic volatility generalising Hobson-Rogers, Working paper.

[14] Kawai, R. (2007) Adaptive Monte Carlo variance reduction with two-time-scale stochastic approximation, Monte Carlo Methods and Applications, 13(3) 197-217.

[15] Platania, A., Rogers, L.C.G. (2003) Putting the Hobson-Rogers model to the tests, Preprint.

[16] Sekine, J. (2008) Marginal distributions of some path-dependent stochastic volatility model, to appear in Statistics \& Probability Letters.

[17] Takeuchi, A. (2008) The Bismut-Elworthy-Li type formulae for stochastic differential equations with jumps, Preprint. 

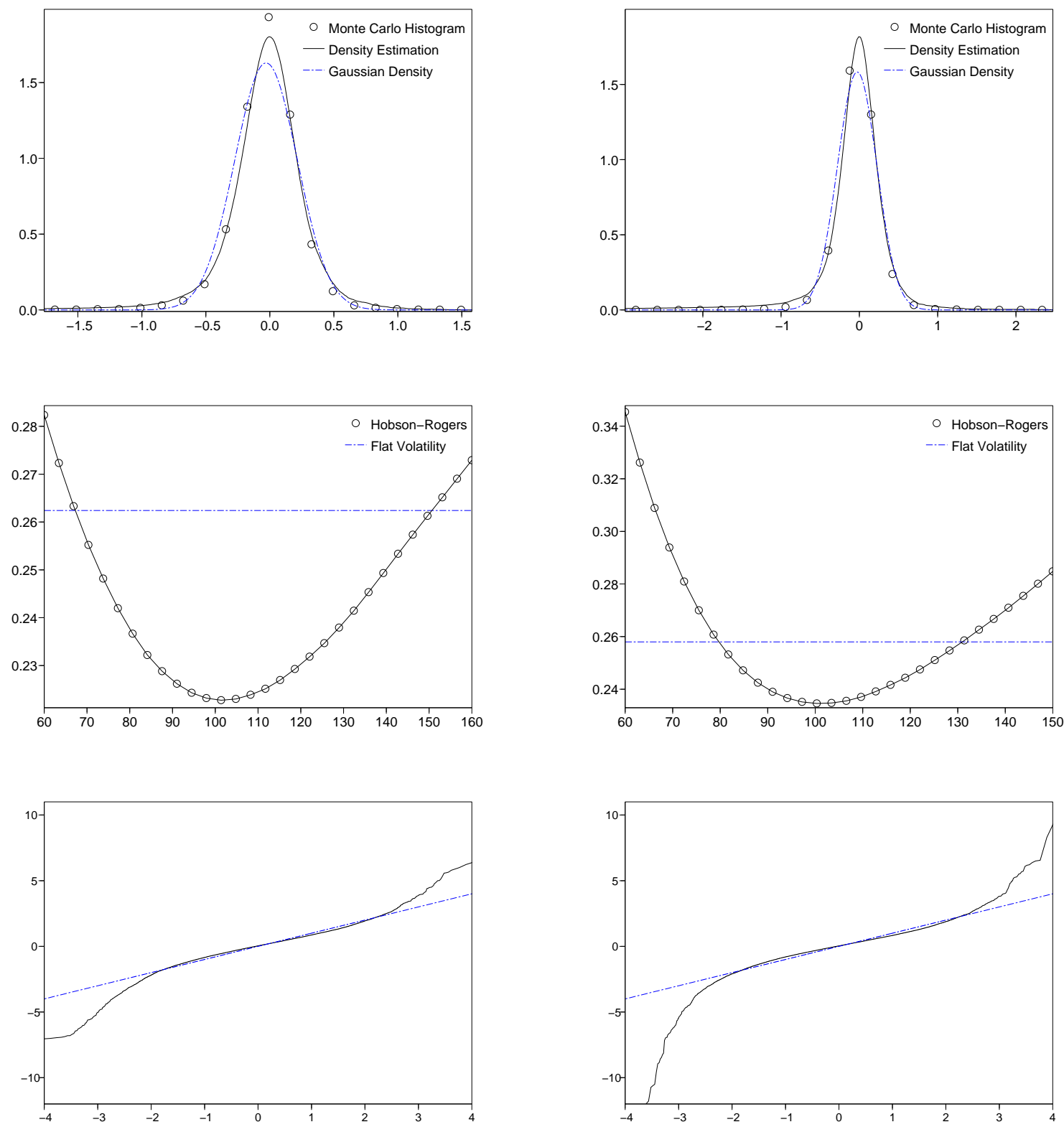

Figure 2: Estimated Density (top), induced implied volatility curve (middle), and QQ-plot (bottom); the three figures on the left are of $\sigma_{1}(y)=0.2\left(1+100 y^{2}\right)^{1 / 2} \wedge N$, while those on the right are of $\sigma_{2}\left(y_{1}, y_{2}, y_{3}\right)=0.2\left(1+\max \left(0,100 y_{1}^{2}+5 y_{3}\right)\right)^{1 / 2} \wedge N$. 